Der Beitrag ökonomischer Bewertung im Rahmen des Flusseinzugsgebietsmanagements

\title{
Von Nutzen und Transfers
}

\author{
Das Flusseinzugsgebietsmanagement zielt auf einen interdisziplinären Ansatz. \\ Gemäß dem Anspruch der europäischen Wasserrahmenrichtlinie spielt dabei die \\ ökonomische Dimension eine wichtige Rolle. Hierbei sollte es nicht nur um Ko- \\ sten, sondern auch um Nutzen gehen. Für eine umfassende Bewertung stehen \\ verschiedene ökonomische Methoden zur Verfügung, die kritisch hinsichtlich \\ ihrer Eignung zu prüfen sind.
}

$\mathrm{V}$

Von Alexandra Dehnhardt or dem Hintergrund der EU-Wasserrahmenrichtlinie (WRRL) ist zentrales Ziel des Flusseinzugsgebietsmanagements die Entwicklung und Umsetzung geeigneter Strategien für eine nachhaltige Bewirtschaftung von Flussgebieten. Dieser Anspruch erfordert

- auf der einen Seite eine Abwägung bezüglich der Folgewirkungen verschiedener Managementstrategien und

- auf der anderen Seite die Entwicklung eines gemeinsamen Verständnisses im Hinblick auf die Probleme und Prozesse, die mit der Umsetzung der Strategien aus Sicht unterschiedlicher Disziplinen verbunden sind.

Ökonomischen Analyse- und Bewertungsansätzen kann dabei in zweierlei Hinsicht Bedeutung zugemessen werden: erstens explizit im Hinblick auf die wirtschaftliche Analyse im Sinne der WRRL. Hiermit sollen die Informationsgrundlagen bereitgestellt werden, um einerseits das Kostendeckungsprinzip bei der Wasserpreisgestaltung zu berïcksichtigen und dabei Anreize für eine effiziente Wassernutzung zu schaffen. Andererseits soll damit die Kosteneffizienz von Maßnahmeprogrammen bewertet werden können.

Entsprechend der gegenwärtigen umweltpolitischen Praxis werden umweltverbessernde Maßnahmen nahezu ausschließlich kostenorientiert gesehen. Das zentrale Entscheidungskriterium der Verhältnismäßigkeit von Kosten verzichtet bislang auf eine (monetäre) Nutzenbewertung, der Nutzen drückt sich vielmehr implizit in den definierten Umweltzielen aus. Daher können zweitens mit der Bewertung des Nutzens umweltverbessernder Maßnahmen Schutzinteressen in einer (Kosten/Nutzen-orientierten) politischen Entscheidungsfindung repräsentiert werden.

Damit gewinnt eine integrierte ökologisch-ökonomische Bewertung auch für das Management von Flusseinzugsgebieten potenziell an Bedeutung. Die ökonomische Analyse ist damit in der Lage, mehr zur Erreichung von Umweltzielen zu leisten, als auf den ersten Blick in der WRRL vorgesehen.

\section{- Die ökonomische Dimension der WRRL}

Mit Blick auf die expliziten Anforderungen der WRRL erfolgt die Integration ökonomischer Bewertung im Wesentlichen durch drei Elemente:

1. die wirtschaftliche Analyse der Wassernutzungen nach Art. 5 (für die Sektoren Landwirtschaft, Industrie und Haushalte),

2. die Deckung der Kosten der Wasserdienstleistungen (Art. 9) und

3. Ausnahmetatbestände (Art. 4).

Im Zuge der wirtschaftlichen Analyse werden die Datengrundlagen zur Berechung kostendeckender Wasserpreise sowie zur Schätzung von Maßnahmekosten geschaffen, um die in Bezug auf die Wassernutzung kosteneffizienten Maßnahmenprogramme identifizieren zu können. Handelt es sich bei der wirtschaftlichen Analyse primär um eine Bestandsaufnahme, besteht Interpretationsbedarf im Hinblick auf die Anforderungen der beiden anderen Elemente. Mit der Erhebung kostendeckender Wasserpreise spielen umweltökonomische Aspekte eine wesentliche Rolle. Durch die ausdrücklich geforderte Einbeziehung von Umwelt- und Ressourcenkosten wird auf eine gesamtwirtschaftliche Perspektive und den mit der Wassernutzung zusammenhängenden Umwelt- und Ressourcenverbrauch abgehoben. Mit der entsprechenden, das Verursacherprinzip zugrundelegenden (kostendeckenden) Wasserpreisgestaltung sollen gleichzeitig angemessene Anreize für eine effiziente Wassernutzung geschaffen werden. Diese Ansprüche betonen eine umweltökonomische, volkswirtschaftliche Perspektive sowie deren Wertansätze: die externen Kosten der Wassernutzung sollen im Grundsatz in die Preisgestaltung einbezogen werden, das heißt ihre vollständige Erfassung ist erforderlich (1). Dies eröffnet neue Perspektiven für die ökonomische Naturbewertung.

Als Ausnahmetatbestände werden so genannte heavily modified water bodies, also erheblich veränderte Gewässer, ausgewiesen. Bei diesen müssen - vereinfacht ausgedrückt - mit einer Maßnahmenumsetzung aufgrund unverhältnismäßiger Kosten für die Wassernutzung geringere Umweltziele erreicht werden. Damit ist ein weiter Interpretationsspielraum gegeben, auf welcher Ebene und aufgrund welcher Kriterien eine Bewertung der Unverhältnismäßigkeit stattfinden soll (2). Aus volkswirtschaftlicher Sicht kann die Frage der Verhältnismäßigkeit nicht ohne eine Bewertung der Nutzen einer Maßnahmenumsetzung beantwortet werden. Beispielsweise ist die Renaturierung eines Fließgewässers im Vergleich mit den Kosten, die sich für die gegenwärtige Wassernutzung - zum Beispiel durch die Landwirtschaft - ergeben würden, unter Umständen mit einem höheren Nettonutzen für die Gesellschaft verbunden.

\section{Ansatzpunkte ökologisch- ökonomischer Bewertung}

Flusssysteme stellen eine Reihe von Gütern und Leistungen zur Verfügung, die mit einem Wert für die Gesellschaft verbunden sind. Im Rahmen der Bewirtschaftungsplanung von Flussgebieten ist eine Entscheidung darüber zu treffen, welche Bewirtschaftungsalternative mit der höchsten Nutzenstiftung - beziehungsweise mit den geringsten Opportunitätskosten - verbunden ist, um eine optimale Allokation der knappen Ressourcen zu gewährleisten.

Die Bereitstellung von Grundlagen zur Abwägung zwischen verschiedenen Optionen ist zentrale Aufgabe der ökologisch-ökonomischen Bewertung. Dabei wird nicht der Wert eines Flusssystems als solcher, sondern lediglich Veränderungen im Hinblick auf das Ausmaß der bereitgestellten Werte betrachtet. Gemäß der neoklassischen (umwelt-) ökonomischen Theorie basiert die Bewertung auf der Ermittlung individueller Präferenzen und letztlich einer aggregierten Wertschätzung für die jeweiligen Umweltgüter. Aus umweltökonomischer Sicht lassen sich bei der Bewertung verschiedene Wertkategorien unterscheiden, die auf der Funktionalität von Ökosystemen und den entsprechend zur Verfügung gestellten Gütern und Leistungen beruhen (vgl. Abb. 1 auf S. 24). 
Abb. 1: Stellenwert ökonomischer Bewerłung im Rahmen des Flussgebietsmanagements

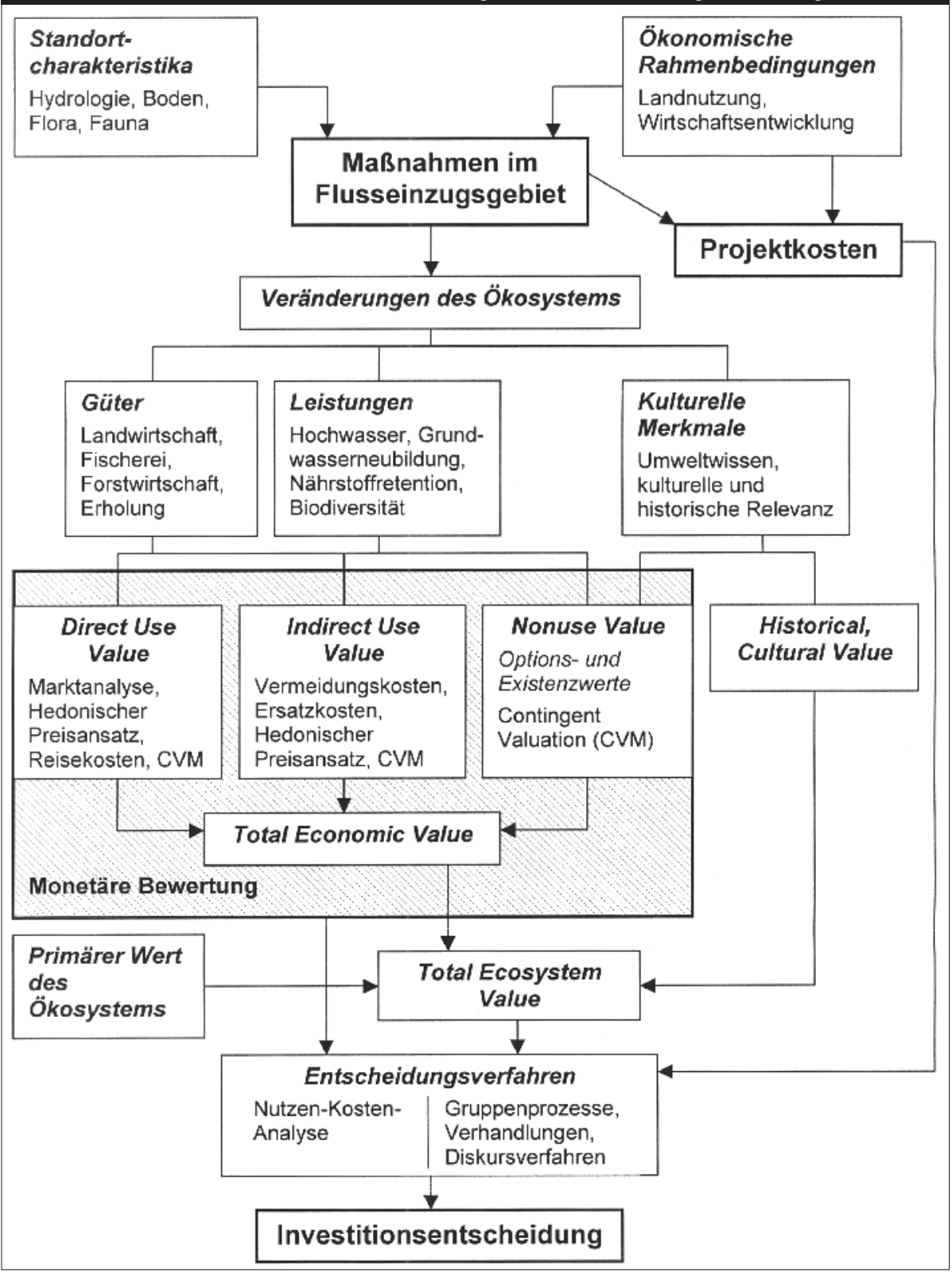

Quelle: deutlich verändert nach Turner, R.K. et al.: Ecosystem Functions and Services: An Integrated Framework and Case Study for Environmental Evaluation. CSERGE Working Paper GEC 2000-21, S. 2

Ein wesentlicher Bestandteil des gesamten Wertes von Ökosystemen (total ecosystem value) ist der ökonomische Gesamtwert (total economic value), der für eine Bewertung von Naturveränderungen mit verschiedenen methodischen Ansätzen erfasst werden kann. Bei den so genannten use values handelt es sich um Werte, die direkt über die Nutzung einer Ressource gewonnen werden. Die indirect use values lassen sich aus der Funktionalität, also den ökologischen Leistungen, die von Ökosystemen bereitgestellt werden, ableiten. Non-use values basieren hingegen auf der Existenz von Ökosystemen, ohne dass diese (aktuell) unmittelbar genutzt werden (3).
Dominierende Verfahren zur Identifizierung dieser Werte sind: der hedonische Preisansatz, die Reisekostenmethode und die Contingent Valuation Method (CVM). Setzen die beiden erstgenannten Ansätze an der Messung der Nutzenwerte durch Ableitung der Wertschätzung aus beobachtetem Verhalten von Individuen an, so versucht die CVM zugleich die non-use values anhand der Erfassung einer hypothetischen Zahlungsbereitschaft durch direkte Befragung festzustellen. Funktionale Werte von Ökosystemen, das heißt die vielfältigen ökologischen Leistungen, werden in der Regel mit so genannten objektiven Bewertungsmethoden erfasst. Diese legen zum Beispiel die Kosten eines technischen Substitutes zur Be- reitstellung derselben Funktion oder aber die Kosten zur Vermeidung eines Schadens bei Wegfall der Leistung zugrunde.

Neben den mit den dargestellten Methoden messbaren ökonomischen Nutzen für die Gesellschaft haben Ökosysteme aufgrund ihrer Fähigkeit zur Selbstorganisation, Stabilität und insbesondere $R \boldsymbol{e}$ silienz - also der Reorganisation und Funktionserhaltung nach externen Störungen - als life support systems einen Wert als solches für den Erhalt der Funktionalität eines Flusssystems. Aus systemökologischer Perspektive liegt darin der primäre Wert eines Ökosystems. Daneben existieren kulturelle oder historische Werte, die als intangible Nutzen ebenfalls ökonomisch nicht zu erfassen sind. Die Anforderungen an einer nachhaltigen Entwicklung verpflichtete Managementstrategien beziehen sich immer auch auf die Aufrechterhaltung der Resilienz von Systemen, da ökonomische (monetär messbare) Werte auf dieser Kapazität beruhen.

\section{Bewertungsgrenzen}

Die Anwendung dieser Bewertungsansätze ist auf der einen Seite mit einer Vielzahl von methodischen Problemen verbunden, auf der anderen Seite kann kritisch diskutiert werden, ob eine ökonomische Bewertung im dargestellten Sinne für das Flussgebietsmanagement überhaupt ein problemadäquater Ansatz sein kann. Aus der Perspektive der Ökologischen Ökonomie ergibt sich eine entscheidende Begrenzung in der Aussagekraft des auf der Erfassung individueller Präferenzen beruhenden (neoklassischen) Bewertungsparadigmas, da nicht alle Werte, die ökologische Systeme für die Gesellschaft haben, über Präferenzen gemessen werden können. Dies gilt insbesondere für die primären Werte. Gleichzeitig wird in diesem Zusammenhang die instrumentelle Bewertung von Ökosystemen kritisiert. Allerdings kann dem entgegnet werden, dass basierend auf dem ökonomischen Erklärungsanspruch zumeist implizit vorhandene Bewertungen lediglich entscheidungsrelevant gemacht werden. Ohne den intrinsischen Wert zu vernachlässigen kann damit eine Monetarisierung durch das Aufzeigen der ökonomischen Relevanz ökosystemarer Leistungen zur Transparenz von Entscheidungen beitragen.

Weiter kann argumentiert werden, dass eine ökonomische Bewertung dann nicht hinreichend ist, wenn die Wertbildung - und damit die Möglichkeit Präferenzen auszudrücken - erst als Ergebnis sozialer Prozesse zu verstehen ist. Eine ökonomische Bewertung kann in diesem Sinne nicht alleinige Grundlage für politische Entscheidungen sein, sondern bedarf 
vielmehr einer weiteren Interpretation im Rahmen der Wertbildung und gesellschaftlicher Willensbildungsprozesse. Die entscheidende Frage bei der Suche nach geeigneten Ansätzen kann daher auch nicht ausschließlich methodisch sein, sondern erfordert gleichzeitig eine Diskussion über die Bedeutung eines Wertes und die Nutzbarkeit von Bewertungsergebnissen im politischen Kontext.

Die skizzierten Grenzen der ökonomischen Bewertung von Naturgütern ebenso wie deren teilweise bislang nur unzureichend gelöste methodische Schwierigkeiten sind keineswegs trivial. Von kritischer Seite wird betont, dass es trotz vergleichsweise intensiver Forschung in diesem Bereich bislang nicht gelungen ist, alle ökosystemaren Funktionen einer ökonomischen Bewertung zuzufuihren, um auf dieser Grundlage den total economic value zu bestimmen. Dieses Konzept sei mithin lediglich ein theoretisches Konstrukt, aber kein für die politische Praxis tauglicher Ansatz. Häufig wird bei exemplarischen Studien nur auf einzelne Komponenten fokussiert und lediglich eine einzelne Funktion eines Ökosystems bewertet, etwa die Nährstoffretention in Flussauen. Oder aber es wird ein mehr oder weniger aggregiertes Bündel an Maßnahmen und entsprechenden Veränderungen im Hinblick auf die Umweltgüter betrachtet, ohne dass einzelne Werte differenziert werden können - ein Problem, das häufig mit kontingenten Bewertungsstudien einhergeht.

\section{- Was eine ökonomische Bewertung leisten kann}

Eine ökonomische Bewertung kann dennoch einen wertvollen Beitrag in mehrerer Hinsicht leisten. Mit dem Flusseinzugsgebietsmanagement wird im Sinne des Ökosystemmanagements ein neuer Politikansatz versucht, der ganz wesentlich an einer Rückbesinnung auf die natürlichen Lebensgrundlagen ansetzt. Eine ökonomische Bewertung dieser Lebensgrundlagen kann auf der einen Seite Argumente dafür liefern, dass ökologisch orientierte Maßnahmen sich auch ökonomisch lohnen können. Gleichzeitig liefert sie einen Beitrag zur Verbesserung der Wahrnehmbarkeit ökologischer Leistungen und unterstiitzt damit die Bewusstseins- und Wertebildung von Stakeholdern. Mit den Ansätzen ökologisch-ökonomischer Bewertung wird darüber hinaus versucht, der Langfristigkeit von Nutzen Rechnung zu tragen und ihnen damit gegenüber den meist kurzfristig wirksamen Kosten ein stärkeres Gewicht im Entscheidungskalkuil zu geben.

Damit kann sie auch einen wesentlichen Beitrag zur Umsetzung der Ziele der WRRL leisten. Zu diesem
Zweck muss jedoch ein vereinfachtes methodisches Instrumentarium geschaffen werden, da sich bisherige Bewertungsstudien als ausgesprochen zeit- und kostenintensiv dargestellt haben. Ein potenziell für die Planungspraxis im Rahmen des Flussgebietsmanagements nutzbares, kosteneffektives Instrument kann der so genannte benefit transfer (Nutzentransfer) sein. Im Grundsatz zielt dieser Ansatz darauf ab, Ergebnisse bereits durchgeführter Studien zur ökonomischen, auf monetären Werten basierenden Bewertung auf andere Anwendungsfälle, das heißt in andere geographische als auch kulturelle Zusammenhänge zu übertragen. Dieser Ansatz scheint vor allem dann geeignet, wenn die Notwendigkeit der Exaktheit gegenüber der Wahrnehmung bzw. der Abschätzung von Größenordnungen im Rahmen von Nutzen-Kosten-Analysen eine weniger große Rolle spielt. Brouwer (4) gibt einige Kriterien an, die die Basis für einen sinnvollen Gebrauch darstellen. Diese stellen die Bedeutung der Stakeholder in den Mittelpunkt, vor allem bei der Definition von Leistungen, der Identifikation von stakeholder values im Hinblick auf die ökonomische Bewertung und deren Akzeptanz. Mit diesem Vorgehen wird zudem eine enge Verbindung zwischen der Methode des Nutzentransfers und der Akzeptanz dieser Bewertung im Zusammenhang mit partizipativen Ansätzen hergestellt. Es erscheint daher gerade für die verschiedenen Ansprüche im Rahmen des Flusseinzugsgebietsmanagement geeignet.

\section{Anmerkungen}

(1) Kommission der Europäischen Gemeinschaft: Die Preisgestaltung als politisches Instrument zur Förderung eines nachhaltigen Umgangs mit Wasserressourcen. Brüssel 2000. (2) Dieser Frage ist das IÖW im Rahmen einer ökonomischen Analyse und Bewertung der deutschen Fallstudien eines EU-Projektes zur "Identifikation und Ausweisung 'erheblich veränderter Wasserkörper' im Sinne der EG-Wasserrahmenrichtlinie" für die Universitüt Kassel bzw. das Umweltbundesamt näher nach gegangen.

(3) Vgl. z.B. Turner, R.K. et al. : Ecological-economic analysis of wetlands: Scientific integration for management and policy. In: Ecological Economics, Vol. 35 (2000), S. 7-23. (4) Brouwer, R.: Environmental value transfer: state of the art and future prospects. In: Ecological Economics. Vol. 32 (2000), S. 137-152.

\section{Die Autorin}

Alexandra Dehnhardt ist wissenschaftliche Mitarbeiterin im Forschungsfeld Umweltökonomie und -politik des Instituts für ökologische Wirtschaftsforschung (IÖW).

Kontakt: IÖW, Potsdamer Str. 105, 10785 Berlin. Tel. 030/884594-27, Fax 030/ 8825439,

E-mail: Alexandra.Dehnhardt@ioew.de

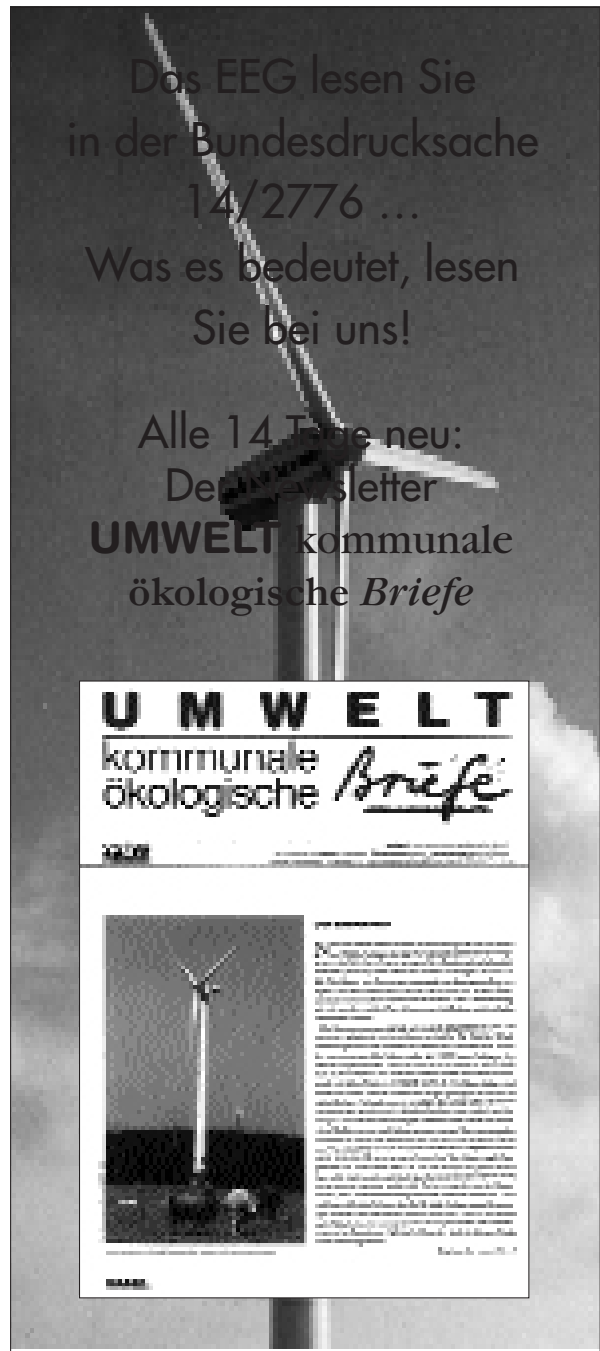

Fordern Sie gleich ein kostenloses Probeabo an!

Schicken Sie den Coupon bitte an:

UmweltBriefe, Raabe Fachverlag

Kaiser-Friedrich-Str. 90, D-10585 Berlin

Fon 030/21 2987-23 Fax 030/21 2987-20

E-mail UMWELTBRIEFE@raabe.de

Internet http://www.umweltbriefe.de

Ja, senden Sie mir die nächsten drei Ausgaben von UMWELT kommunale ökologische Briefe kostenlos zu. Wenn Sie eine Woche nach Erhalt des letzten Freiexemplares nichts von mir hören, bestelle ich bis auf weiteres, mindestens aber für ein Jahr, UMWELT kommunale ökologische Briefe. Der Jahresbezugspreis für ein Abonnement (26 Ausgaben) beträgt 360 DM (unverb. Preisempf.) zzgl. Versandspesen.

Name:

Straße:

PLZ, Ort:

Datum:

Unterschrif*):

Ich bin/wir sind berechtigt, die Bestellung des Abonnements ohne Angaben von Gründen innerhalb einer Woche nach Erhalt der drei Freiexemplare in schrifflicher Form zu widerrufen. Zur Wahrung der Frist genügt die rechtzeitige Absendung des Widerrufs (Poststempel).

Datum: Unterschrift*): *) Bitte an beiden Stellen unterschreiben 
(c) 20I0 Authors; licensee IÖW and oekom verlag. This is an article distributed under the terms of the Creative Commons Attribution Non-Commercial No Derivates License (http://creativecommons.org/licenses/by-nc-nd/3.o/), which permits unrestricted use, distribution, and reproduction in any medium, provided the original work is properly cited. 